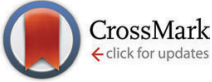

Cite this: Phys. Chem. Chem. Phys., 2015, 17, 11053

Received 26th December 2014, Accepted 20th March 2015

DOI: $10.1039 / c 4 c p 06070 a$

www.rsc.org/pccp

\section{Optical properties of irradiated imidazolium based room temperature ionic liquids: new microscopic insights into the radiation induced mutations $\dagger$}

\author{
Apurav Guleria,* Ajay K Singh and Soumyakanti Adhikari
}

Considering the future perspectives of room temperature ionic liquids (RTILs) in areas involving high radiation fields (such as the nuclear fuel cycle and space applications), it is essential to probe and have a microscopic understanding of the radiation induced perturbations in the molecular structures and the intrinsic bonding interactions existing in the ILS. Herein, a focused investigation concerning the photophysical behavior of postirradiated FAP (fluoroalkyl phosphate) imidazolium ILs revealed considerable rearrangements and bonding realignments of the ionic moieties in the ILs on irradiation, however, their physicochemical properties do not change significantly even at high absorbed doses. Most interestingly, the well-established excitation wavelength dependent fluorescence (FL) behavior of the ILs was considerably perturbed on irradiation and this is attributed to the radiation induced decoupling of pre-existing different associated structures of ions, and the subsequent formation of oligomers and other species containing multiple bond order groups. This was further substantiated by vibrational studies, where peaks appearing in the range $1600-1800 \mathrm{~cm}^{-1}$ indicated the formation of double bonded products. Furthermore, for the hydroxyl functionalized (in the alkyl side chain of the imidazolium cation) IL, a blue shift in the $\mathrm{O}-\mathrm{H}$ stretching frequency was observed for the $-\mathrm{OH}$ group $\mathrm{H}$-bonded to the FAP anion $\left(\nu \mathrm{OH} \cdots[F A P]^{-}\right)$, while a red shift was observed for the $\mathrm{H}$-bonded $-\mathrm{OH}$ groups in the cationic clusters. The FL lifetime values were found to increase with irradiation, which clearly indicates the enhancement in the rigidity level in the vicinity of the ions, thereby hindering the non-radiative decay processes. Such studies could contribute to the fundamental understanding of the radiation driven perturbations in the structure-property relationships, which eventually affect the radiolytic degradation pathways and the product distribution in RTILs.

\section{Introduction}

Photophysical studies of imidazolium room temperature ionic liquids (RTILs) have been extensively carried out in recent times, and they show unconventional behavior of such solvents. ${ }^{1 a-e}$ Optical spectroscopic studies of neat imidazolium based RTILs have already been carried out by Samanta et $a l^{1 d-g}$ and other researchers. ${ }^{2 a, b}$ In these studies, an unusual excitation wavelength dependent fluorescence behavior (known as the red edge

Radiation \& Photochemistry Division, Bhabha Atomic Research Centre, Mumbai 400 085, India. E-mail: aguleria@barc.gov.in; Fax: +91-22-25505331; Tel: +91-22-25596116

$\dagger$ Electronic supplementary information (ESI) available: Plots of fluorescence intensity $v s$. dose and $\lambda_{\mathrm{em}} v s$. dose for FAP1 and FAP2 at different excitation wavelengths are shown. Discussion related to the vibrational analysis of the postirradiated FAP ILs is provided along with their spectra. Tables showing the corresponding $\lambda_{\mathrm{em}}$ and maximum PL intensity values for neat pre- and postirradiated FAP ILs at $\lambda_{\text {exc. }}$ and absorbed dose values are shown. Average lifetime values of neat unirradiated and post-irradiated FAP ILs at various absorbed doses are provided. See DOI: 10.1039/c4cp06070a effect or REE or REES) was reported for these media. REES is defined as a shift in the wavelength of the maximum fluorescence emission towards higher wavelengths, caused by a shift in the excitation wavelength towards the red edge of the absorption band. REES is generally observed in microheterogeneous media and highly viscous solutions. Essentially, this phenomenon arises from the slow rates of solvent relaxation (reorientation) around an excited state fluorophore, which is a function of the motional restriction imposed on the solvent molecules in the immediate vicinity of the fluorophore. The excitation near the peak or on the blue side of an electronic absorption tends to excite a broad distribution of energetically different solutesolvent environments. In contrast, excitation on the red edge of an absorption band preferentially selects a narrowed and redshifted subset of the overall distribution. ${ }^{2 c}$ Nevertheless, in ILs, excitation wavelength dependent fluorescence behavior has been attributed to the presence of various associated species and the inefficiency of the excitation energy-transfer between them or an incomplete solvation process. ${ }^{1-g, 2}$ In fact, recently, Ghosh et $a .^{2 a}$ reported the heterogeneity of the fluorescence lifetime of 
FAP (fluoroalkyl phosphate) based imidazolium RTILs, which clearly indicated the absence of any excited state phenomenon (like solvation and energy transfer) in these fluids. Essentially, ILs are microheterogeneous in nature with a parallel co-existence of different types of interactions (such as coulombic, dipolar, van der Waals and H-bonding), which make these fluids unique and complex in comparison to conventional solvents. ${ }^{3}$ Keeping this in mind, information regarding the photophysical behavior of irradiated ILs could be useful in gaining a better understanding of the radiation driven perturbations in the structure-property relationships, which eventually affect the radiolytic degradation pathways and the product distribution. Moreover, RTILs, owing to their unique physicochemical properties, have aroused a lot of interest in recent times for their potential application in areas involving high radiation fields, such as nuclear fuel reprocessing, space and other industrial applications. ${ }^{4}$

In this pretext, numerous radiation stability studies on RTILs (especially imidazolium based ones) including important reviews are available in the literature. ${ }^{5}$ Allen et al. ${ }^{5 a}$ have primarily assessed the effects of $\alpha$-, $\beta$ - and $\gamma$-radiation on hydrophilic RTILs, such as 1-butyl-3-methyl imidazolium nitrate ([BMIM] $\mathrm{NO}_{3}$ ), 1-ethyl3-methyl imidazolium chloride ([EMIM]Cl) and 1-hexyl-3-methyl imidazolium chloride ([HMIM $] \mathrm{Cl})$. These authors reported that $\sim 1 \%$ of the samples undergo radiolytic degradation up to an exposure dose of $400 \mathrm{kGy}$. Qi et al. and Yuan et al. investigated the influence of $\gamma$-radiolysis on imidazolium based fluoroanions, such as $\left(\left[\mathrm{PF}_{6}\right]^{-}\right)$, and tetrafluoroborates $\left(\left[\mathrm{BF}_{4}\right]^{-}\right)$using spectroscopic methods along with differential scanning calorimetry (DSC). ${ }^{5 b-d}$ Dhiman et al. ${ }^{5 e}$ investigated the radiolytic production of molecular hydrogen in aromatic and aliphatic ILs irradiated with $\gamma$-rays, protons and helium ions to determine the functional dependence of the yield (of hydrogen) on the particle track structure. It was found by these researchers that the hydrogen gas yield increases considerably on going from low linear energy transfer (LET, defined as the energy deposition per unit length) radiations ( $\gamma$-rays) to high LET (protons and helium ions) radiations in case of aromatic ILs, while the same remain essentially constant for aliphatic ILs. Intratrack chemistry of highly excited states has been assigned as the most probable reason for the increase in the hydrogen gas yield for aromatic ILs when irradiated with high LET radiation. However, for aliphatic ILs, the LET effect was found to be insignificant, ${ }^{5 e}$ as the $\mathrm{H}$ atom abstraction and combination reactions are mainly responsible for the production of $\mathrm{H}_{2}$. Berthon and co-workers ${ }^{5 f, g}$ examined the radiation stability of hydrophobic imidazolium based ILs containing anions such as hexafluorophosphate $\left(\left[\mathrm{PF}_{6}\right]^{-}\right)$, bis(trifluoromethylsulfonyl) imide $\left(\left[\mathrm{NTf}_{2}\right]^{-}\right)$and $[\mathrm{N} 1444]\left[\mathrm{NTf}_{2}\right]$ where $[\mathrm{N} 1444]^{+}$is the methyl tributylammonium cation. These authors proposed a possible degradation scheme of the RTILs based on NMR and mass studies. The effect of commonly used anions, e.g. $\left[\mathrm{NTf}_{2}\right]^{-}$, trifluoromethanesulfonate $\left([\mathrm{TfO}]^{-}\right),\left[\mathrm{PF}_{6}\right]^{-}$and $\left[\mathrm{BF}_{4}\right]^{-}$on the radiochemical stability of imidazolium based ILs under $\gamma$-irradiation was investigated by

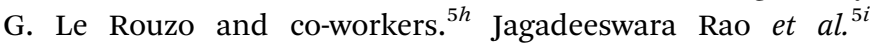
reported the influence of radiation on the rheometric and electrochemical properties of some halide and $\mathrm{NTf}_{2}$ based ILs. Yuan et al. ${ }^{5 j}$ observed radiation induced darkening of ionic liquid [BMIM] $\left[\mathrm{NTf}_{2}\right]$ and the subsequent decoloration on adding different oxidants. The majority of these investigations clearly show insignificant variations in the physicochemical properties of ILs (especially imidazolium based ones) on irradiation to high absorbed doses. Some researchers have also conducted fluorescence and vibrational studies to probe the radiolytic degradation and mechanism of the ILs. ${ }^{5,6}$ For example, Qi et al. ${ }^{5 b}$ reported the fluorescence and the Raman spectra of $\gamma$-irradiated IL, $[\mathrm{BMIM}]\left[\mathrm{PF}_{6}\right]$. Radiation induced significant chemical scissions of the $n$-butyl group (e.g. $\mathrm{C}-\mathrm{H}$ and $\mathrm{C}-\mathrm{C}$ scission) as well as damage to the $\left[\mathrm{PF}_{6}\right]^{-}$anion. FTIR measurements reported by Yuan et $a{ }^{5 c}{ }^{5}$ showed signs of a slight acceleration of the radiolysis of [BMIM] $\left[\mathrm{PF}_{6}\right]$ in the presence of nitric acid. The Raman spectral studies by Qi et $a .^{5 d}$ indicated that the radiolysis of $[\mathrm{BMIM}]\left[\mathrm{BF}_{4}\right]$ induces a detectable destruction of the alkyl chain and scission of the $\mathrm{C}-\mathrm{H}$ bonds in the ring of the imidazolium cation, and relatively small changes in the anion. Recently, Dhiman et $a .^{5 e, l}$ carried out gamma and heavy ion beam irradiation of aromatic and aliphatic ILs. Primarily, $\mathrm{H}_{2}$ gas yield measurements of the post-irradiated ILs were conducted by these researchers, which were found to be consistent with the findings from the FTIR and UV-Vis analysis. All these studies indicate the necessity of detailed studies focusing on the photophysical behavior (such as fluorescence and vibrational studies) of the post-irradiated imidazolium ILs.

Therefore, in this report, an exclusive investigation concerning the photophysical behavior of FAP based imidazolium ILs before and after irradiation was conducted in conjunction with elaborate vibrational spectroscopic studies (FTIR and Raman) for the comprehensive understanding of the radiation induced structural changes. Most interestingly, the well established excitation wavelength dependent behavior of imidazolium ILs was considerably perturbed on irradiation. Moreover, the fluorescence lifetime values measured for the neat ILs were found to increase with irradiation, and the trend was more pronounced for the hydroxyl group functionalized IL. From the vibrational studies, interesting aspects were revealed, such as significant molecular rearrangements of the ions and realignments of the bonding interactions in the irradiated ILs.

The structures of the ILs studied in this manuscript are shown in Fig. 1. It is to be noted that the ILs, 1-ethyl-3-methylimidazolium tris(pentafluoroethyl)trifluorophosphate ([EMIM][FAP]) and 1-(2-hydroxyethyl)-3-methylimidazolium tris(pentafluoroethyl)trifluorophosphate ([EOHMIM][FAP]), have been abbreviated to FAP1 and FAP2, respectively, in this manuscript.

\section{Experimental}

\subsection{Chemicals}

FAP1 (1-ethyl-3-methylimidazolium tris(pentafluoroethyl)trifluorophosphate, [EMIM][FAP]), and FAP2 (1-(2-hydroxyethyl)-3methylimidazolium tris(pentafluoroethyl)trifluorophosphate, [EOHMIM][FAP]), were obtained from Merck KGaA, Darmstadt, Germany, with a purity $>99 \%$. Since these ILs are highly hydrophobic and the water uptake was extremely small, they were used as 


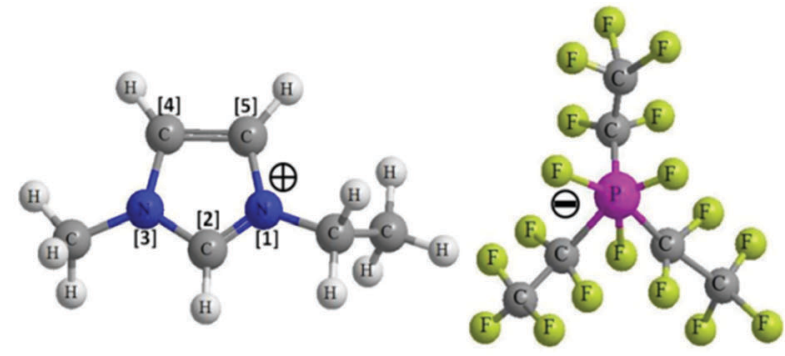

(a) [EMIM][FAP]

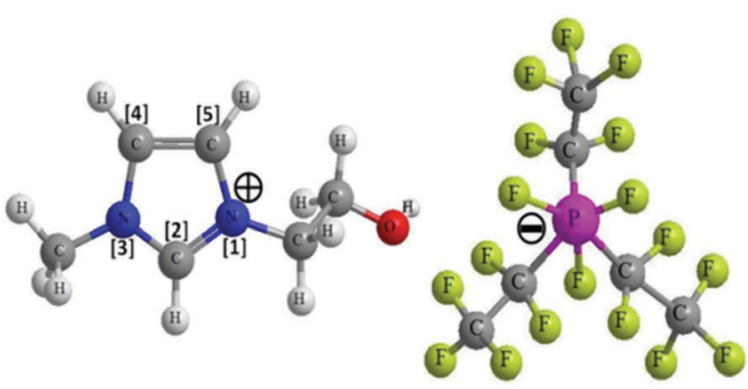

(b) [EOHMIM][FAP]

Fig. 1 Structures of the FAP based imidazolium ILs studied.

received without further processing. Nonetheless, the water contents in the as studied FAP ILs, i.e. FAP1 and FAP2, were determined and found to be $30 \mathrm{ppm}$ and $55 \mathrm{ppm}$, respectively, by the coulometric Karl Fischer method using a Metrohm 831 KF Coulometer.

\subsection{Irradiation of the ILS}

The ILs were irradiated with a $7 \mathrm{MeV}$ electron beam (FWHM $\sim 2 \mu \mathrm{s}$ ) obtained from a linear accelerator (LINAC) described elsewhere. ${ }^{7}$ The absorbed dose was measured using an air-saturated solution containing $5 \times 10^{-2} \mathrm{~mol} \mathrm{dm}^{-3} \mathrm{KSCN}$ assuming $\mathrm{G} \varepsilon$ for $(\mathrm{SCN})_{2}{ }^{--}=$ $2.6 \times 10^{-4} \mathrm{~m}^{2} \mathrm{~J}^{-1}$ at $475 \mathrm{~nm}^{8}$ The absorbed dose per pulse was maintained at $160 \mathrm{~Gy}$ and the samples were irradiated with repeated pulses at 50 pulses per second, accounting for a cumulative dose ranging from 10 to $400 \mathrm{kGy}$.

\subsection{Instrumentation}

UV-Vis optical absorption studies were carried out on a JASCO V-650 spectrophotometer at room temperature. Prior to recording the UV-Vis absorption spectra, all the samples were diluted with acetonitrile to avoid the saturation level (observed using both $10 \mathrm{~mm}$ and $1 \mathrm{~mm}$ path lengths) occurring due to the intense color that appeared on irradiating the FAP ILs. Acetonitrile was used as a diluent because of its miscibility with the FAP ILs and its negligible absorption above $200 \mathrm{~nm}$. Photoluminescence (PL) measurements were recorded on a Hitachi F-4500 spectrofluorimeter at room temperature. Emission life time measurements were conducted on a time correlated single photon counting (TCSPC) instrument (model: IBH, UK). The instrument response function (IRF) of the setup was measured by collecting the scattered light from a $\mathrm{TiO}_{2}$ suspension in water. The samples were excited at $394 \mathrm{~nm}$ by a laser source with an IRF of less than 150 ps (full width at half maximum, FWHM) and the decay traces were monitored at an emission wavelength of $500 \mathrm{~nm}$. The optical path length of the cell in all the spectral studies was $10 \mathrm{~mm}$. The FT-IR spectra were recorded using a diamond single reflectance ATR probe in an IR Affinity-1 spectrometer. Raman spectra of the samples were recorded using a STR-300 micro-Raman spectrometer (SEKI Technotron, Japan). The sample solutions were excited at $532 \mathrm{~nm}$, sourced from a fibre coupled diode-pumped solid-state laser (DPSS). The scattered light was collected through a fiber-coupled $300 \mathrm{~mm}$ spectrograph (Acton series SP 2300i, $1200 \mathrm{~g} \mathrm{~mm}^{-1}$ ) and detected by a thermo-electric cooled $\left(-75^{\circ} \mathrm{C}\right)$ charge-coupled device (CCD).

\section{Results and discussion}

On irradiation, the colorless and transparent ILs darkened with the increase in absorbed dose. The UV-Vis absorption spectra of pre- and post-irradiated FAP2 at various absorbed doses are shown in Fig. 2. The picture showing the color evolution in the same IL on irradiation at various doses is provided in the inset of Fig. 2. The absorption spectra and the color evolution for FAP1 were reported earlier. ${ }^{9}$

It can be seen that the absorption spectra of the irradiated ILs showed a peak at $\sim 300 \mathrm{~nm}$ accompanied by a shoulder at $\sim 370 \mathrm{~nm}$. The intensity of the former peak increased, while the later one slightly red shifted with the increase in absorbed dose. The radiolytic products of the imidazolium cation (especially, the oligomeric species of imidazole and its derivatives) were found to be primarily responsible for the color evolution and the emergence of peaks in the absorption spectra of the irradiated FAP ILs, as reported in our earlier work. ${ }^{9}$ The red shift in the shoulder peak (with the increase in absorbed dose) could most

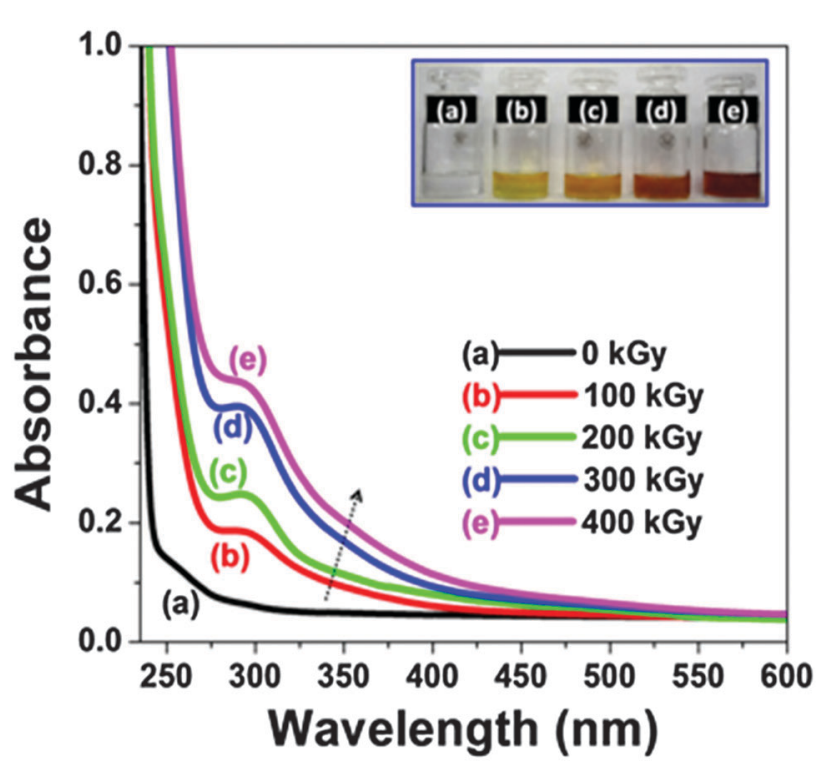

Fig. 2 UV-Vis absorption spectra of pre- and post-irradiated FAP2 at various absorbed doses in kGy. Inset: picture showing the color change of FAP2 on irradiation. Doses in kGy: (a) 0, (b) 100, (c) 200, (d) 300 and (e) 400 . 
plausibly be the indication of the formation of higher order oligomeric species. These trends and observations are in agreement with those reported by various groups on other imidazolium based ILs containing various anions such as $\left[\mathrm{NTf}_{2}\right]^{-},,^{-5, g}\left[\mathrm{PF}_{6}\right]^{-5 b, c, f}$ and $\left[\mathrm{BF}_{4}\right]^{-5 d}$

Most interestingly, the well established excitation wavelength dependent fluorescence behavior of imidazolium ILs was found to be considerably perturbed on irradiation. The emission spectra of pre- and post-irradiated FAP1 at different levels of absorbed dose are shown in Fig. 3. Fig. S1 (ESI $\dagger$ ) shows the emission spectra of FAP1 irradiated at $400 \mathrm{kGy}$. As can be seen from Fig. 3A, the unirradiated IL shows two emission bands (at $\sim 340 \mathrm{~nm}$ and a shoulder peak at $\sim 450 \mathrm{~nm}$ ) when excited at the lower wavelength region. However, as the excitation wavelength is increased, the two emission bands merge into a single broad peak with a simultaneous bathochromic shift.

Surprisingly, there was subsequent quenching of the fluorescence at lower excitation wavelengths after irradiation to different degrees depending on the absorbed doses (see Fig. 3 and Fig. S2a, ESI $\dagger$ ). At the same time, the maximum emission peaks showed bathochromic shifts for the higher excitation wavelengths with the gradual increase in the absorbed dose (Fig. S2b, ESI $\dagger$ ). Such corresponding trends can also be envisaged from the plots of $\lambda_{\text {exc. }} v s$. $\lambda_{\text {em }}$, shown in the insets of the emission spectra of irradiated FAP1 IL (Fig. 3). For a better understanding, the maximum fluorescence intensity and $\lambda_{\mathrm{em}}$ values corresponding to the respective excitation wavelengths and absorbed doses for neat FAP1 (pre- and post-irradiated) are tabulated in Table S1 $(\mathrm{ESI} \dagger)$. It is worth noting that Qi et $a l^{5 b}$ have previously reported the fluorescence spectra (at only one $\lambda_{\text {exc. value of }}$ $290 \mathrm{~nm}$ ) of $\gamma$-irradiated IL [BMIM] $\left[\mathrm{PF}_{6}\right]$ (diluted with dimethylformamide, DMF), which showed the appearance of peaks between 300 to $600 \mathrm{~nm}$, similar to the present case.

Analogous to FAP1, FAP2 containing the -OH group displayed similar excitation wavelength dependent behavior after irradiation (see Fig. S3, ESI $\dagger$ ). However, with FAP2, the decrease

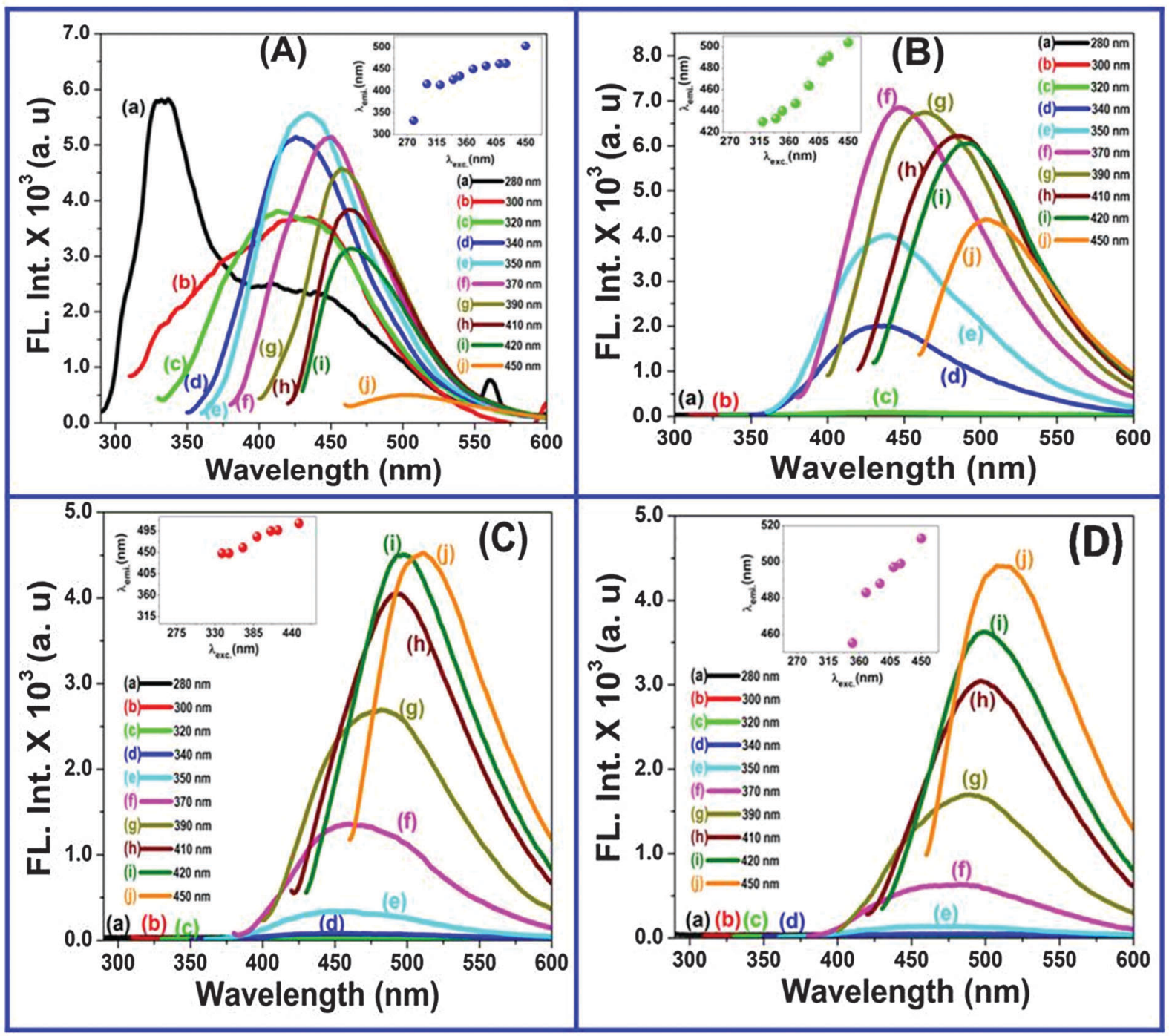

Fig. 3 Fluorescence spectra of neat unirradiated (A) and post-irradiated FAP1 at various absorbed doses, i.e. 100 kGy (B); 200 kGy (C); and 300 kGy (D). Insets: plots of $\lambda_{\text {exc. }} v s$. $\lambda_{\text {em }}$ showing the shifts in the maximum intensity peak positions. 
in the fluorescence intensity as well as the red shift in the emission peaks were more pronounced for the subsequent increase in the excitation wavelengths at higher absorbed doses. These trends can be visualized in Fig. S4 and Table S2 (ESI $\dagger$ ). Considering the excitation wavelength of $450 \mathrm{~nm}$ and the absorbed dose of $400 \mathrm{kGy}$, it can be seen that the red shift (with respect to the unirradiated IL) in the emission peak was $18 \mathrm{~nm}$ for FAP1, while it was $60 \mathrm{~nm}$ for FAP2 (see Fig. S5, ESI $\dagger$ ). These observations indicate the formation of higher order (in terms of conjugation and introduction of new $\pi-\pi$ interactions) radiolytic products in FAP2. Nevertheless, as already mentioned, the excitation wavelength dependent fluorescence spectra of imidazolium ILs are attributed to the existence of various associated structures of the cations and anions. ${ }^{1,2}$ In fact, some of the associated forms of the cations and anions were observed during the mass spectrometric studies of neat unirradiated FAP1 and FAP2. ${ }^{9}$ For instance, ions with $m / z$ values of 667.1 ([(EMIM) FAP $\left.]^{+}\right)$and $699.1\left(\left[(\mathrm{EOHMIM})_{2} \mathrm{FAP}\right]^{+}\right)$were noticed in the mass spectra of unirradiated FAP1 and FAP2, respectively. However, these ions were found to be very weak in nature since a collision energy of only $2 \mathrm{eV}$ led to their dissociation into the respective parent ions. Therefore, the as-described perturbations in the excitation wavelength dependent behavior of the irradiated FAP ILs could be attributed to the irradiation induced decoupling or the break down of such pre-existing different associated forms of the cations and anions (in the unirradiated IL), simultaneously driving the formation of oligomers and other species containing multiple bond order groups. Indeed, this was substantiated by the vibrational studies (FTIR and Raman), which are discussed below.

There are very few vibrational studies (FTIR and Raman) of irradiated ILs in the literature and they are not in much detail. Interestingly, we have noticed a strong correlation between the vibrational changes and fluorescence behavior of the irradiated ILs. The FTIR spectra of pre- and post-irradiated FAP1 are shown in Fig. S6 (ESI $\dagger$ ), while Fig. 4 shows the FTIR spectra in the particular wavenumber regions with significant alterations on an amplified scale for better visibility. Some of the discussion regarding the changes in the peak positions and their intensities (on irradiation of FAP1) is provided in the ESI. $\dagger$
Up to a dose of $100 \mathrm{kGy}$, not many changes could be observed in the FTIR spectra of the irradiated ILs, which reflects their radiation stability. However, at a high absorbed dose of $400 \mathrm{kGy}$, some modulations were observed as follows. The peak at $\sim 890 \mathrm{~cm}^{-1}$ (see Fig. $4 \mathrm{a}$ and peak marked as [1] in Fig. S6, ESI $\dagger$ ) is attributed to olefinic $\mathrm{C}-\mathrm{H}$ bending vibrations, which indicates the formation of radiolytic products with a vinylidene double bond $\left(=\mathrm{C}=\mathrm{CH}_{2}\right) .{ }^{10}$ Another absorption band centered at $\sim 1736 \mathrm{~cm}^{-1}$ (see Fig. $4 \mathrm{~b}$ and peak marked as [2] in Fig. S6, ESI $\dagger$ ) signifies the formation of species with double bonds in the form of $\mathrm{C}=\mathrm{C},-\mathrm{CF}=\mathrm{CF}-$, and $-\mathrm{CF}=\mathrm{CF}_{2}{ }^{11}$ It has been reported earlier ${ }^{9}$ that the amount of radiolytic products formed on irradiation of FAP ILs is small (i.e. $<5 \%$ of the parent ion peak), however, we have been able to identify some of the possible radiolytic products possessing double bond units from their mass spectrometric studies. The plausible molecular structures of these products are shown in Fig. S7 (ESI $\dagger$ ), along with the mass spectra of post-irradiated FAP2. Similar types of products were noticed in the case of the irradiated FAP1 IL. $^{9}$ Moreover, recently, Wang et al., ${ }^{12 a}$ by means of UV-Vis analysis and density functional theory calculations, proposed that the color darkening in imidazolium based IL $[\mathrm{BMIm}]\left[\mathrm{NTf}_{2}\right]$ upon irradiation originates from the formation of double bonds in the aliphatic chains of pristine organic cations (or radiolytic products of RTILs) and various associated species containing these "double bond products". Therefore, our vibrational analysis provides direct evidence for the formation of aforesaid radiolytic products containing double bonds, which eventually might act as chromophoric units. Besides this, strong evidence has been reported by various researchers for the existence of hydrogen bonding interactions in ILs (especially imidazolium based ILs) on the basis of spectroscopic (mainly X-ray diffraction, IR and NMR spectroscopy) and computational studies. ${ }^{12}$ For example, shorter $\mathrm{C}-\mathrm{H}$ anion distances, red shifted $\mathrm{C}-\mathrm{H}$ stretching frequencies and downfield shifted $\mathrm{C}-\mathrm{H}$ proton chemical shifts were the observed indications of hydrogen bonding in imidazolium based ILs. ${ }^{12 e}$ Furthermore, it has been observed in earlier studies $^{12 d, e}$ that the hydrogen bonding interactions involving the $\mathrm{C}(4)-\mathrm{H}$ and $\mathrm{C}(5)-\mathrm{H}$ groups are weaker compared to those

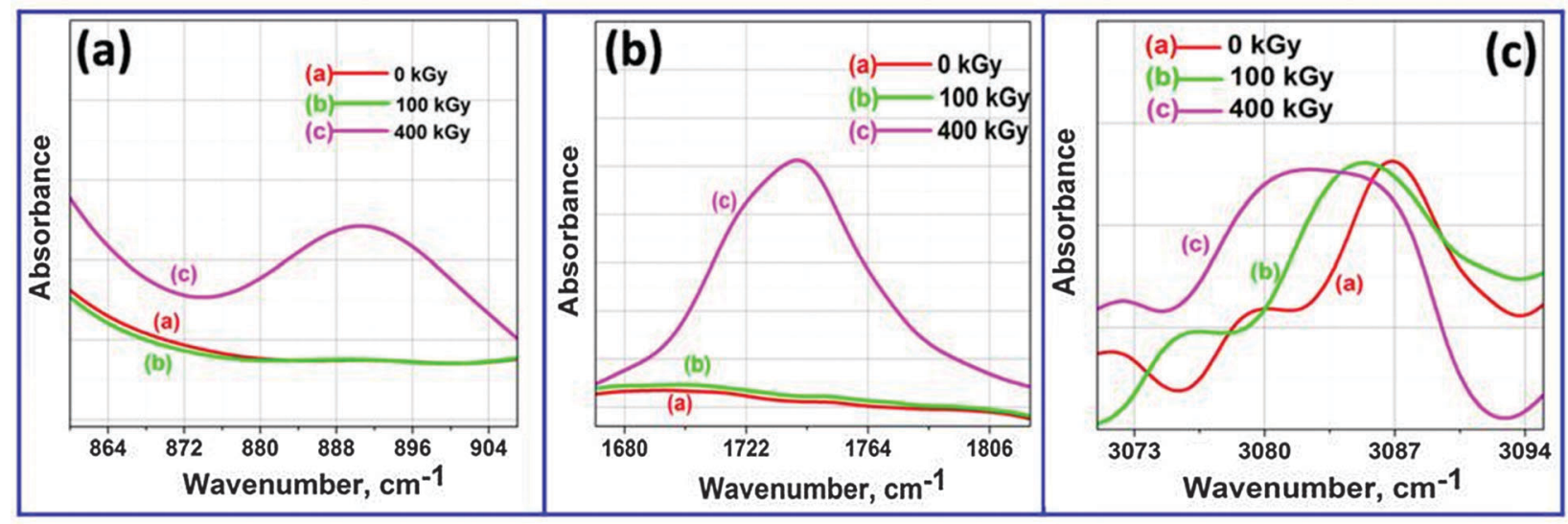

Fig. 4 FTIR spectra of neat unirradiated and post-irradiated FAP1 in different wavenumber regions at various absorbed doses, i.e. 0 kGy, 100 kGy and 400 kGy. 
involving the $\mathrm{C}(2)-\mathrm{H}$ groups. A stronger NMR downfield proton chemical shift of about $1 \mathrm{ppm}$ for $\mathrm{C}(2)-\mathrm{H}$ compared to $\mathrm{C}(4,5)-\mathrm{H}$ has been reported. ${ }^{12 d}$ In agreement with this, quantum-chemical calculations (e.g. for the IL $[\mathrm{BMIM}]\left[\mathrm{PF}_{6}\right]$ in the gas phase) have also suggested stronger hydrogen bonding interactions between the $\mathrm{C} 2$ proton and the anion, compared to those between the $\mathrm{C}(4,5)$ protons and the anion. ${ }^{12 f}$ On the whole, the $\mathrm{C}-\mathrm{H}$ bond of the imidazolium cation carries important information about the existence and strength of the hydrogen bonding network in the RTILs. However, substantial variations in the $\mathrm{C}-\mathrm{H}$ bond stretching frequencies (in the region $2000-3000 \mathrm{~cm}^{-1}$ ) of the ethyl group and the terminal methyl group attached to the side chain of the imidazolium cation were observed and this is discussed in the ESI. $\dagger$ Furthermore, a peak at $\sim 3086 \mathrm{~cm}^{-1}$ (for unirradiated FAP1), originating from $(\mathrm{N}) \mathrm{CH}_{3} \mathrm{HCH}$ asymmetric stretching vibrations, ${ }^{13,14}$ was red shifted on irradiation of the IL (shown in Fig. 4c). Subsequently, the broadening of the peaks signifies the presence of radiolytic products mostly derived from the imidazolium cation with varying $\mathrm{C}-\mathrm{H}$ bond strengths. Moreover, a red shift observed in the ring $\mathrm{NC}(\mathrm{H}) \mathrm{NCH}\left(\right.$ or $\left.\mathrm{C}_{2}-\mathrm{H}\right)$ vibrational frequency from $3130 \mathrm{~cm}^{-1}$ to $3126 \mathrm{~cm}^{-1}$ on irradiation of FAP1 clearly shows weakening of the respective bond strength. ${ }^{5 e}$ Certainly, these variations substantiate the realignment of the intermolecular hydrogen bonding interactions among the ionic moieties in the case of the post-irradiated FAP1 IL. Furthermore, it has been reported that the $\mathrm{C}-2$ position in the imidazolium cation is acidic $\left(\mathrm{p} K_{\mathrm{a}}=21-22\right)^{15}$ in nature and prone to deprotonation to form a stabilized carbene. Therefore, the weakening of the bond strength of the $\mathrm{C}_{2}-\mathrm{H}$ bond on irradiation can be regarded as strong evidence supporting the vulnerability of imidazolium-derived radical species to undergo oligomerization. ${ }^{5,9,16}$ Consequently, this has been found to be one of the possible contributing factors behind the color evolution in the irradiated imidazolium ILs.

The FTIR spectra of post-irradiated FAP2 at different absorbed doses (same as for FAP1) are shown in Fig. S8 and S9 $(\mathrm{ESI} \dagger)$. Analogous to FAP1, the formation of radiolytic products with multiple bond order functional groups as well as bathochromic shifts in the $\mathrm{C}-\mathrm{H}$ stretching frequencies were also observed for the post-irradiated FAP2 IL (see ESI $\dagger$ ). Furthermore, a broad shoulder centered at $\sim 3430 \mathrm{~cm}^{-1}$ was observed in addition to a peak at $\sim 3621 \mathrm{~cm}^{-1}$ for unirradiated FAP2 (see Fig. 5). These peaks were also observed in the Raman spectra of unirradiated FAP2, as shown in the inset of Fig. 5. A similar type of broad feature at $\sim 3425 \mathrm{~cm}^{-1}$ and a peak at $\sim 3598 \mathrm{~cm}^{-1}$ have been reported recently by Katsyuba et $a l .{ }^{17}$ for $[\mathrm{EOHMIM}]\left[\mathrm{PF}_{6}\right]$. The former peak is assigned to the $\nu \mathrm{OH}$ of hydrogen bonded cationic clusters, while the later one is attributed to the stretching frequency of $\mathrm{OH}$ hydrogen bonded to the $\mathrm{F}$ atom of the $\mathrm{PF}_{6}$ anion $\left(\nu \mathrm{OH} \cdots\left[\mathrm{PF}_{6}\right]^{-}\right)$. The $\nu \mathrm{OH}$ for the isolated [EOHMIM] $]^{+}$cation has been reported to be $3690 \mathrm{~cm}^{-1}$ by computational analysis. ${ }^{17}$ Taking account of these observations, the peak at $\sim 3621 \mathrm{~cm}^{-1}$ (observed in the present work) is attributed to the stretching frequency of - $\mathrm{OH}$ hydrogen bonded to the FAP anion $\left(\operatorname{Im}(\mathrm{OH}) \cdots[\mathrm{FAP}]^{-}\right)$. The broad shoulder centered at $\sim 3430 \mathrm{~cm}^{-1}$ can be assigned to the stretching frequency of $-\mathrm{OH}$ hydrogen bonded to other hydroxyl groups of neighboring cations; the preexistence of such cationic clusters has been observed and discussed earlier. The as-discussed - $\mathrm{OH}$ bonding interactions possibly existing in FAP2 have been pictorially represented in Fig. 5 .

Nevertheless, an interesting observation of a blue shift in the peak frequency from $\sim 3621 \mathrm{~cm}^{-1}$ (for unirradiated FAP2) to $\sim 3634 \mathrm{~cm}^{-1}$ (in post-irradiated FAP2) was noticed (Fig. 5). This signifies the weakening of the hydrogen bond $\left(\operatorname{Im}(\mathrm{OH}) \cdots[\mathrm{FAP}]^{-}\right)$ between the $\mathrm{OH}$ and the FAP anion. On the other hand, the broad peak at $\sim 3430 \mathrm{~cm}^{-1}$ was red shifted to $\sim 3380 \mathrm{~cm}^{-1}$, indicating the reduction in the $\mathrm{O}-\mathrm{H}$ bond strength. This points towards a possible distribution of the $\mathrm{O}-\mathrm{H}$ bond strength, which could be due to the involvement of the $-\mathrm{OH}$ group in multiple non-covalent bonding interactions with the newly formed radiolytic species in its neighborhood. At this stage, it is important to mention that the geometry involving the orientation and the interactions of the cations and the anions in the $-\mathrm{OH}$ group functionalized IL is different from that of the non-hydroxyl IL. Recently, the interactions of $[\mathrm{EOHMIM}]^{+}$and $[\mathrm{EMIM}]^{+}$with


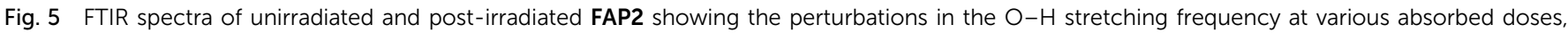

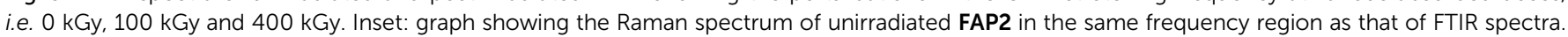
A pictorial representation of the $-\mathrm{OH}$ bonding interactions possibly existing in FAP2 is shown along with the graph. 
various anions were investigated using DFT calculations and NMR spectroscopy by Zhang et al. ${ }^{18}$ According to the optimized geometries obtained, the anions were located in front of the imidazolium ring, where the atoms carrying more negative charges were closer to the proton at the C-2 position in the non-hydroxyl ILs. However, the anions were somewhat above the imidazolium ring, moving close to the $-\mathrm{OH}$ group in the case of hydroxyl containing ILs, such as FAP2. Considering this, the aforementioned shifts in the vibrational peaks can be regarded as important evidence for the reorientation or restructuring occurring in FAP2 on irradiation.

Raman spectra of the irradiated FAP ILs further substantiate the FTIR studies. Baseline corrected Raman spectra (Fig. S10 and S11, ESI $\dagger$ ) of neat FAP1 and FAP2 indicate the perturbations and re-organization in the IL lattice on irradiation (see ESI $\dagger$ ). A red shift was observed in the symmetric bending modes of $-\mathrm{CF}_{3}$ (at $\sim 742 \mathrm{~cm}^{-1}$ ) for FAP1 on irradiation (see Fig. 6a). Furthermore, a broad peak centered at $\sim 985 \mathrm{~cm}^{-1}$ appeared in the Raman spectra of both the FAP ILs (see Fig. S12a and S13b, ESI $\dagger$ ) on irradiation (dose $\geq 50 \mathrm{kGy}$ ), which indicates the formation of radiolytic products with vinyl groups. ${ }^{10}$ An important observation was the appearance of peaks in the range $1600-1650 \mathrm{~cm}^{-1}$ in both the irradiated FAP ILs and they have been assigned to the formation of radiolytic products with conjugated $\mathrm{C}=\mathrm{C}$ groups (alkenyl $\mathrm{C}=\mathrm{C}$ stretching). ${ }^{10}$ This could be regarded as another strong piece of evidence for the formation of chromophoric species responsible for the color evolution in imidazolium based ILs on irradiation. Recently, Dhiman et al. ${ }^{5 l}$ also reported the appearance of a peak at $1658 \mathrm{~cm}^{-1}$, which was attributed to the formation of acyclic di-substituted alkene bonds in the irradiated imidazolium based ILs.

The vibrational studies hitherto categorically reflect the considerable changes in the inherent spatial correlations of the ionic species (in the ILs) on irradiation. NMR $\left({ }^{1} \mathrm{H}\right.$ and $\left.{ }^{19} \mathrm{~F}\right)$ spectra of both the FAP ILs, before and after irradiation, were recorded to examine the amount and the mechanism of radiolytic degradation, and this was reported in our earlier work. ${ }^{9}$ The ${ }^{1} \mathrm{H}$ NMR spectra of the irradiated ILs (especially FAP1) showed small changes in the chemical shift values and the emergence of some small peaks compared to the unirradiated ones. The slight chemical shifts in the peaks indicate perturbation in the bonding rearrangements of the ionic moieties. For instance, the chemical shift values of the C-2 proton in the ${ }^{1} \mathrm{H}-\mathrm{NMR}$ spectra of both the FAP ILs decreased on irradiation. Furthermore, the broadening and the decrease in the chemical shift value of the peak corresponding to the hydroxyl group proton of the irradiated FAP2 are attributed to the variations in the hydrogen bond between the hydroxyl group of the cation and the fluorine atom of the FAP anion $(\mathrm{OH} \cdots \mathrm{F})$. The decreases in the chemical shift values of the C-2 proton and the hydroxyl proton are evidence for the alterations in the distribution and the nature of the interactions accessible to the aforementioned protons due to the formation of some new species, on radiolysis of the ILs. Similar variations, pointing towards the radiation induced realignment of the ionic moieties in the ILs, have been reported in earlier work. ${ }^{9}$ Indeed, these observations further substantiate the findings (regarding the radiation driven internal molecular restructuring of the ILs) of the present photophysical studies on the post-irradiated FAP ILs.

The influence of irradiation induced decoupling of pre-existing particular arrangements and related perturbations in the bonding interactions (amongst cationic and anionic moieties) were also indicated from the fluorescence lifetime behavior of FAP ILs (shown in Fig. 7), especially in the case of FAP2 containing the -OH group. On irradiation, the average lifetime of FAP1 and FAP2 increases with the rise in the absorbed dose imparted, however, the trend was found to be more prominent in the later case (see Table S3, ESI $\dagger$ ). The multi-exponential behavior signifies the heterogeneous nature of these media and indicates the existence of energetically distributed aggregates with different conformations, as has been reported earlier. ${ }^{1,2}$ Nonetheless, the exact reason for the increasing trend in the average lifetime values of the FAP ILs with higher absorbed doses is not precisely clear. However, the most probable explanation could be the
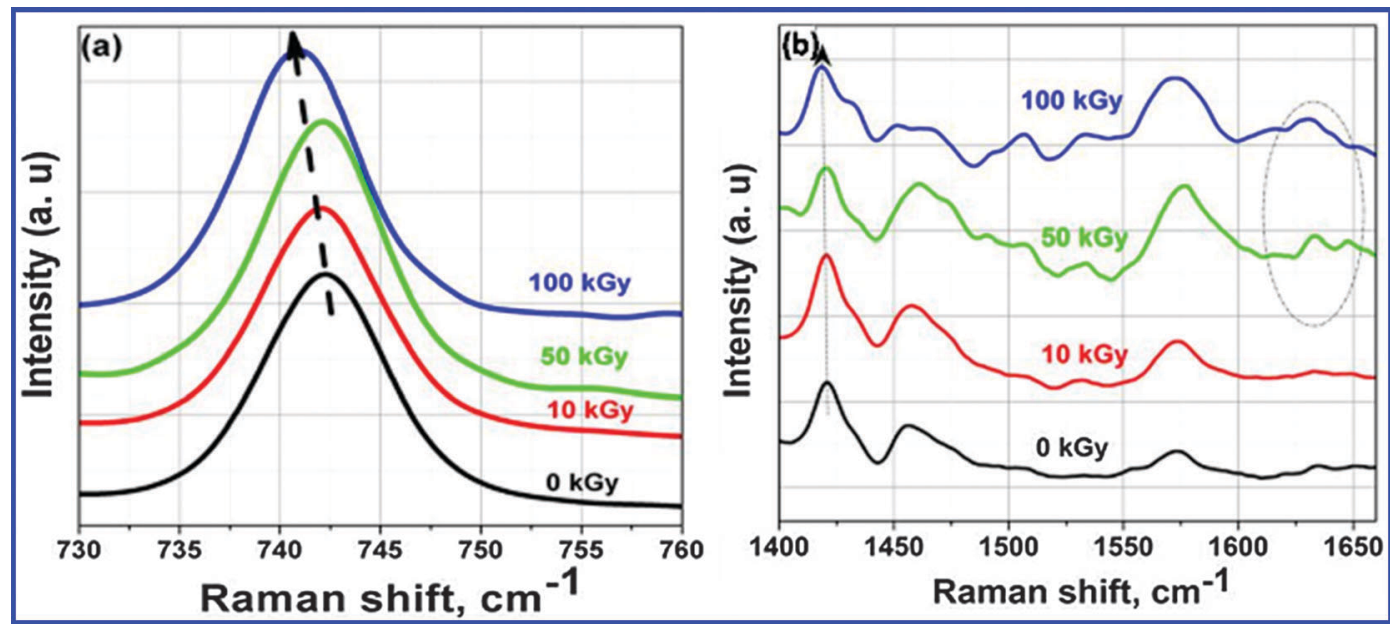

Fig. 6 Raman spectra of neat unirradiated and post-irradiated FAP1 in different wavenumber regions at various absorbed doses, i.e. 0 kGy, 10 kGy, $50 \mathrm{kGy}$ and $100 \mathrm{kGy}$. 




Fig. 7 Lifetime decay profile of neat irradiated FAP1 (a) and FAP2 (b) at various absorbed doses.

formation of new intermolecular hydrogen bonds and other noncovalent bonding interactions (e.g. $\pi-\pi)$ between the radiolytic products and the ionic moieties, as indicated from the vibrational studies.

Consequently, enhancement in the rigidity level in the vicinity of the ions (especially the imidazolium cation, which is primarily responsible for the fluorescence behavior of imidazolium based ILs) can be anticipated, which eventually might hinder the non-radiative decay processes leading to the increase in the average lifetime values observed for the post-irradiated FAP ILs.

\section{Conclusions}

The physicochemical properties of FAP based imidazolium ILs (especially the non-hydroxyl IL) were found to vary insignificantly on irradiation with high absorbed doses, ${ }^{9}$ however, the photophysical behavior of the pre- and post-irradiated FAP ILs categorically points towards considerable changes in the orientations and the molecular rearrangements of the ions on irradiation. For instance, (i) bathochromic shifts in the excitation wavelength dependent fluorescence spectra of the irradiated FAP ILs (due to the irradiation induced break down of pre-existing different associated structures of ions and subsequent formation of various radiolytic products including oligomers), (ii) vulnerability of $\mathrm{C}_{2}-\mathrm{H}$ to undergo deprotonation (indicated by the red shift in the vibrational frequency), (iii) a blue shift in the $\mathrm{O}-\mathrm{H}$ stretching frequency (for the $-\mathrm{OH}$ functionalized FAP2 on irradiation), and (iv) the observation of vibrational peaks originating due to multiple bond order groups (which could be a contributor to the color evolution in the post-irradiated imidazolium IL) are some of the highlights of this study, which could be useful in gaining a comprehensive understanding of the radiation driven degradation of ILs and their mechanistic pathways. We believe that the present study will be helpful in gaining a microscopic insight into the radiation induced mutations in the structure-property relationships and the nature of various interactions existing in ILs.

\section{Acknowledgements}

The authors thank LINAC staff for their technical help and cooperation in carrying out experiments in the LINAC facility of RPCD, BARC. We would like to thank Shri Trilochan Gadly, Dr P. G. Jaison and Mr K. K. Singh for carrying out the Karl Fischer titrations, ESI-mass spectrometry and FTIR measurements, respectively. We would like to acknowledge SAIF, IIT Bombay for allowing us to carry out ESI-MS measurements. We thank Dr J. A. Mondal for the scientific discussions during the vibrational analysis of the irradiated ILs. The authors thank Dr D. K. Palit, Head, RPCD and Dr B. N. Jagatap, Director, Chemistry Group, BARC for their support and encouragement.

\section{References}

1 (a) H. Weingärtner, Angew. Chem., Int. Ed., 2008, 47, 654-670; (b) S. Arzhantsev, H. Jin, G. A. Baker and M. Maroncelli, J. Phys. Chem. B, 2007, 111, 4978-4989; (c) E. Binetti, A. Panniello, L. Triggiani, R. Tommasi, A. Agostiano, M. L. Curri and M. Striccoli, J. Phys. Chem. $B$, 2012, 116, 3512-3518; (d) A. Paul, P. K. Mandal and A. Samanta, J. Phys. Chem. B, 2005, 109, 9148-9153; (e) P. K. Mandal, M. Sarkar and A. Samanta, J. Phys. Chem. $A, 2004,108$, 9048-9053; $(f)$ A. Paul and A. Samanta, J. Chem. Sci., 2006, 118, 335-340; $(g)$ A. Paul, P. K. Mandal and A. Samanta, Chem. Phys. Lett., 2005, 402, 375-379.

2 (a) A. Ghosh, T. Chatterjee and P. K. Mandal, Chem. Commun., 2012, 48, 6250-6252; (b) S. Cha, T. Shim, Y. Ouchi and D. Kim, J. Phys. Chem. B, 2013, 117, 10818-10825; (c) H. Jin, X. Li and M. Maroncelli, J. Phys. Chem. B, 2007, 111, 13473-13478.

3 (a) Y. Wang and G. A. Voth, J. Am. Chem. Soc., 2005, 127, 12192-12193; (b) J. N. A. Canongia Lopes and A. A. H. Pádua, J. Phys. Chem. B, 2006, 110, 3330-3335; (c) L. M. N. B. F. Santos, J. N. C. Lopes, J. A. P. Coutinho, J. M. S. S. Esperanca, L. R. Gomes, I. M. Marrucho and L. P. N. Rebelo, J. Am. Chem. 
Soc., 2007, 129, 284-285; (d) Y. Wang, W. E. I. Jiang, T. Yan and G. A. Voth, Acc. Chem. Res., 2007, 40, 1193-1199; (e) K. Iwata, H. Okajima, S. Saha and H. O. Hamaguchi, Acc. Chem. Res., 2007, 40, 1174-1181.

4 X. Sun, H. Luo and S. Dai, Chem. Rev., 2012, 112, 2100-2128.

5 (a) D. Allen, G. Baston, A. E. Bradley, T. Gorman, A. Haile, I. Hamblett, J. E. Hatter, M. J. F. Healey, B. Hodgson, R. Lewin, K. V. Lovell, B. Newton, W. R. Pitner, D. W. Rooney, D. Sanders, K. R. Seddon, H. E. Sims and R. C. Thied, Green Chem., 2002, 4, 152-158; (b) M. Qi, G. Wu, S. Chen and Y. Liu, Radiat. Res., 2007, 167, 508-514; (c) L. Yuan, J. Peng, M. Zhai, J. Li and G. Wei, Radiat. Phys. Chem., 2009, 78, 737-739; (d) M. Qi, G. Wu, Q. Li and Y. Luo, Radiat. Phys. Chem., 2008, 77, 877-883; (e) S. B. Dhiman, G. S. Goff, W. Runde and J. A. Laverne, J. Phys. Chem. B, 2013, 117, 6782-6788; $(f)$ L. Berthon, S. I. Nikitenko, I. Bisel, C. Berthon, M. Faucon, B. Saucerotte, N. Zorz and P. Moisy, Dalton Trans., 2006, 2526-2534; (g) E. Bossé, L. Berthon, N. Zorz, J. Monget, C. Berthon, I. Bisel, S. Legand and P. Moisy, Dalton Trans., 2008, 924-931; (h) G. Le Rouzo, C. Lamouroux, V. Dauvois, A. Dannoux, S. Legand, D. Durand, P. Moisy and G. Moutiers, Dalton Trans., 2009, 6175-6184; ( $i$ ) C. Jagadeeswara Rao, K. A. Venkatesan, B. V. R. Tata, K. Nagarajan, T. G. Srinivasan and P. R. Vasudeva Rao, Radiat. Phys. Chem., 2011, 80, 643-649; (j) L. Yuan, J. Peng, L. Xu, M. Zhai, J. Li and G. Wei, Radiat. Phys. Chem., 2009, 78, 1133-1136; (k) B. J. Mincher and J. F. Wishart, Solvent Extr. Ion Exch., 2014, 32, 563-583; (l) S. B. Dhiman, G. S. Goff, W. Runde and J. A. LaVerne, J. Nucl. Mater., 2014, 453, 182-187.

6 P. K. Mohapatra, A. Sengupta, M. Iqbal, J. Huskens and W. Verboom, Chem. - Eur. J., 2013, 19, 3230-3238.

7 T. Mukherjee, in Atomic, Molecular and Cluster Physics, ed. S. A. Ahmad, Narosa Publishing House, New Delhi, 1997, pp. 299-316.

8 G. V. Buxton and C. R. Stuart, J. Chem. Soc., Faraday Trans., 1995, 91, 279-281.

9 A. Guleria, A. K. Singh, S. Adhikari and S. K. Sarkar, Dalton Trans., 2014, 43, 609-625.
10 (a) J. Coates, in Encyclopedia of Analytical Chemistry, ed. R. A. Meyers, John Wiley \& Sons Ltd, Chichester, 2000, pp. 10815-10837; (b) G. Socrates, Infrared and Raman Characteristic Group Frequencies: Tables and Charts, John Wiley \& Sons, 2004; (c) L. J. Bellamy, Infrared Spectra of Complex Molecules, Chapman \& Hall, New York, USA, 1975, vol. 1.

11 (a) Q. T. Le, S. Naumov, T. Conard, A. Franquet, M. Müller, B. Beckhoff, C. Adelmann, H. Struyf, S. D. Gendt and M. R. Baklanov, ECS J. Solid State Sci. Technol., 2013, 2, N93-N98; (b) K. P. Huang, P. Lin and H. C. Shih, J. Appl. Phys., 2004, 96, 354-360; (c) X. Gu, T. Nemoto, A. Teramoto, T. Ito and T. Ohmi, J. Electrochem. Soc., 2009, 156, H409-H415.

12 (a) S. Wang, J. Liu, L. Yuan, Z. Cui, J. Peng, J. Li, M. Zhai and W. Liu, Phys. Chem. Chem. Phys., 2014, 16, 18729-18735; (b) A. Elaiwi, P. B. Hitchcock, K. R. Seddon, N. Srinivasan, Y.-M. Tan, T. Welton and J. A. Zora, J. Chem. Soc., Dalton Trans., 1995, 3467-3472; (c) A. Yokozeki, D. J. Kasprzak and M. B. Shiflett, Phys. Chem. Chem. Phys., 2007, 9, 5018-5026; (d) A. Wulf, K. Fumino, D. Michalik and R. Ludwig, ChemPhysChem, 2007, 8, 2265-2269; (e) C. Roth, S. Chatzipapadopoulos, D. Kerlé, F. Friedriszik, M. Lütgens, S. Lochbrunner, O. Kühn and R. Ludwig, New J. Phys., 2012, 14, 105026; (f) J. H. Antony, D. Mertens, T. Breitenstein, A. Dölle, P. Wasserscheid and W. R. Carper, Pure Appl. Chem., 2004, 76, 255-261.

13 J. Kiefer, J. Fries and A. Leipertz, Appl. Spectrosc., 2007, 61, 1306-1311.

14 E. R. Talaty, S. Raja, V. J. Storhaug, A. Dölle and W. R. Carper, J. Phys. Chem. B, 2004, 108, 13177-13184.

15 S. Sowmiah, V. Srinivasadesikan, M. C. Tseng and Y. H. Chu, Molecules, 2009, 14, 3780-3813.

16 I. A. Shkrob, J. Phys. Chem. B, 2010, 114, 368-375.

17 S. A. Katsyuba, M. V. Vener, E. E. Zvereva, Z. Fei, R. Scopelliti, G. Laurenczy, N. Yan, E. Paunescu and P. J. Dyson, J. Phys. Chem. B, 2013, 117, 9094-9105.

18 S. Zhang, X. Qi, X. Ma, L. Lu, Q. Zhang and Y. Deng, J. Phys. Org. Chem., 2012, 25, 248-257. 